\title{
Calidad de los alimentos, estandarización y ferias del agricultor en Costa Rica
}

\section{Food Quality, Standardization and Farmers Markets in Costa Rica}

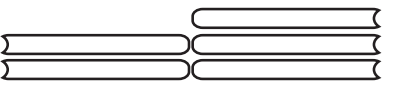

Marianela Zúñiga-Escobar ${ }^{1}$

Paulo André Niederle ${ }^{2}$

Recibido: junio 2016 Aprobado: agosto 2017

\section{Resumen}

El objetivo de este artículo es analizar la perspectiva de la calidad de los alimentos, considerando los procesos de estandarización en el contexto de las ferias del agricultor, siendo éstas un mercado local que contribuye con la seguridad alimentaria y nutricional de la población costarricense. El texto se divide en tres secciones, en la primera se caracteriza el sistema alimentario dominante y el tipo de riesgos alimentarios que presentan los mercados que se encuentran inmersos en éste. En la segunda sección se incluyen argumentos sobre dichos riesgos alimentarios y se discute sobre los estándares de calidad del producto. Éstos, en algunos casos, pueden ser excluyentes porque se realizan bajo condiciones de poca representatividad que responden principalmente al mundo comercial. En la tercera sección se presenta el caso de las ferias del agricultor de Costa Rica, mostrando diversas perspectivas de la calidad que pueden ser valoradas en los mercados locales, tomando en cuenta la importancia de las relaciones sociales y la compra directa que se establece entre sector productor y consumidor.

Palabras clave: Ferias del agricultor, calidad, seguridad alimentaria y nutricional.

Abstract
The aim of this paper is to analyze the perspective of food qual-
ity, considering the standardization processes in the context of
farmer markets, that contributes with food and nutrition secu-
rity of the Costa Rican population. The text is divided into three
sections, the first one is characterized by the dominant food sys-
tem and the type of food risks of those markets involved. The
second section includes information about these food risks and
discussion about the quality of the product standards, which in
de Nutrición, Universidad de Costa Rica. Marianela.zunigaescobaar@ucr.ac.cr


some cases may be exclusive because they are carried out under conditions of poor representation in terms of quality prospects as they belong to the commercial world. The third section presents the farmer markets in Costa Rica showing different quality perspectives that can be valued in local markets, taking into account the importance of social relations and the direct purchase established between the producer-consumer sector.

Keywords: farmer markets, quality, food and nutrition security

\section{Introducción}

A partir de la década de los años 80, varios movimientos de crítica social desencadenaron procesos de reconfiguración de los mercados alimentarios (Goodman, 2003). Una de las implicaciones sobre el tema fue la construcción de "redes alternativas" que abarcan segmentos específicos de productores y consumidores (Goodman, Dupuis e Goodman, 2012). Así, en todo el mundo comenzaron a aparecer en escena las ferias libres, se crearon grupos de consumo ético y responsable, colectivos en pro de los alimentos vegetarianos, asociaciones y cooperativas campesinas, nuevas organizaciones ambientalistas a favor del consumo sustentable-ético-responsable y, recientemente, grupos para la promoción de la agricultura urbana y del consumo local. Estas experiencias buscan crear nuevas trayectorias sociales para los alimentos, articulando mercados en donde además de encontrar bienes alimentarios diferenciados, circulan valores sociales (sustentabilidad, equidad, localidad, artesanía, salubridad) que son la pauta de nuevos modelos de producción y consumo.

Uno de los factores centrales para explicar esos cambios se encuentra en la presión ejercida por un segmento creciente de población consumidora ávida, no solo por el hecho de proveerse de alimentos saludables, ecológicos u orgánicos, sino por algún compromiso frente a la emergencia de la sustentabilidad como nuevo paradigma de la sociedad. En este sentido, destaca la preocupación con el tema de la seguridad alimentaria y nutricional que, además, ha llamado la atención para la revalorización de la sociobiodiversidad asociada al acto de comer. En estas redes alternativas, la alimentación deja de ser solamente una práctica biológica para ser reconceptuada como un acto sociocultural y político, lo cual busca fortalecer las dimensiones de la localidad, de la artesanía, la salubridad y la convivencia.

La legitimación de esos valores llevó a una reacción de los “imperios alimentarios” (Ploeg, 2008) -coordinados por su nuevo actor-líder, el sector 
detallista- con la tentativa continua de la apropiación de las calidades, creación de normas, estándares y certificaciones específicas sobre lo que es la calidad en un determinado alimento. Todo eso para ajustar la demanda de calidades diferenciadas a los mecanismos institucionales que sustentan los modernos regímenes alimentarios (Friedman, 2016; McMichael, 2016).

Estos eventos han permitido la reactivación de nuevos circuitos de comercio. Tal es el caso de las ferias del agricultor, identificadas, hasta hace poco, como un modelo arcaico de relaciones mercantiles cuya extinción inevitable dependería del ritmo de la expansión de las grandes redes de compra al detalle, principalmente de los supermercados. Con una creciente expansión en todo el mundo, estos circuitos directos de producción y consumo (Darlot, 2013; Goodman, 2003) pueden ser vistos como una especie de "contramovimiento" (Polanyi, 1980) a los procesos de mercantilización y convencionalización del sector agroalimentario industrial dominante.

Con el propósito de ampliar la información sobre este tema, el presente artículo tiene como objetivo analizar la perspectiva de la calidad de los alimentos, considerando los procesos de estandarización en el contexto de las ferias del agricultor, siendo éstas un mercado local que contribuye con la seguridad alimentaria y nutricional de la población costarricense. En este contexto, consideramos a las ferias del agricultor, como un ejemplo de un mercado local que promueve la relación directa entre sujetos agricultores y consumidores en Costa Rica, en el cual destacan diversas valoraciones diferenciadas de la calidad, de las cuales el consumidor puede formar parte.

\section{Los riesgos alimentarios en el sistema dominante}

En la actualidad el sistema alimentario dominante ha generado cambios importantes en el tema de consumo de alimentos. Éste es un proceso complejo y multifactorial, que muchas veces se ve afectado por la incoherencia entre lo que se sabe y ofrece como alimentos de calidad y, lo que en la realidad se practica o se tiene alcance, dentro de una oferta bastante robusta.

Desde la década de los años noventa, organismos nacionales e internacionales posicionan con más fuerza la importancia del tema de la seguridad alimentaria y nutricional (SAN), desarrollando definiciones integrales y estrategias de acción para su fortalecimiento. En el contexto Centroamericano, el Instituto de Nutrición de Centroamérica y Panamá (INCAP), institución rectora en el tema en esa región, define la SAN como: "El estado en el que todas las personas disfrutan de forma oportuna y permanente, al acceso de los alimentos 
que necesitan tanto en cantidad y calidad para el consumo adecuado y uso biológico, garantizando un estado de bienestar que contribuye con el desarrollo humano" (INCAP, 2018).

La SAN es un enfoque amplio en el cuál la calidad alimentaria, constituye uno de los factores que determina el consumo y utilización biológica de los alimentos. De acuerdo con nuestra realidad, la Organización Mundial de la Salud (OMS) (2018) hace referencia al tema de inocuidad de alimentos, alertando sobre la presencia de alimentos no saludables que están relacionados con la muerte de cerca de dos millones de personas por año. Además, alerta sobre aquellos alimentos que contienen virus, bacterias, parásitos y otros productos químicos nocivos que causan más de 200 enfermedades que incluyen desde diarreas hasta el cáncer.

La calidad de los alimentos representa una de las características necesarias para garantizar una alimentación adecuada; sin embargo, su efectividad depende de situaciones que han sido transformadas en los últimos tiempos y que limitan el poder de la persona consumidora durante la escogencia de sus alimentos. Algunas tendencias notables de esa transformación han surgido a partir del siglo XX, en donde existían pequeñas propiedades o granjas que se transformaron en grandes corporaciones, que convirtieron los sistemas de producción en unos más intensificados, eficientes y especializados que hoy en día producen para la industria los llamados commodities. Además de eso, se pasó de una sociedad que preparaba los alimentos en casa para una que compra cerca de la mitad de sus comidas preparadas y consumidas en otros lugares, y de una dieta basada en alimentos cultivados localmente para una basada, en gran parte, por alimentos procesados y transportados a largas distancias (Nestle, 2013).

Las corporaciones transnacionales han sido actores centrales en el desarrollo del sistema agroalimentario hegemónico en la actualidad, o a lo que Ploeg (2008) llama como imperios alimentarios en la era de la globalización. Según el autor, estos imperios no representan valor ni producen cualquier valor propio, sino que absorben los valores producidos por otros grupos. Además indica que no poseen ni desenvuelven sus propios recursos independientes, sino que básicamente, usurpan o controlan los recursos de otros; no necesitan de la propiedad directa de una base de recursos y no representan necesariamente valor acumulado.

El poder del imperio alimentario actual se encuentra concentrado en pocas corporaciones; éstas dominan, en la mayoría de los países, la producción y el 
comercio internacional de productos alimentarios y agrícolas. Además, esas corporaciones son actores importantes en el procesamiento, distribución y venta al por menor. Según Goodman y Redclift (1991), debemos hacer una pausa para considerar las consecuencias de este cambio fundamental en el suministro de nuestros alimentos. Dentro de otras cosas, las personas se sienten cada vez más abrumadas con alarmas sobre la contaminación de los alimentos, la pesca excesiva, la tala de los bosques, la pérdida de la biodiversidad, el cambio climático, la contaminación química, las consecuencias potencialmente negativas de los productos modificados genéticamente y muchos otros riesgos ambientales y de salud. Al mismo tiempo, las personas sienten que no pueden confiar únicamente en las autoridades públicas, que han fracasado a menudo para asumir sus responsabilidades (Bostrom y Klintman, 2008).

Según Buchler, Smith y Lawrence (2010), los riesgos en el tema alimentario pueden ser divididos en dos subcategorías: "tradicionales" y "modernos". Los riesgos tradicionales, tienen que ver con la contaminación y deterioro alimentario, las fechas de caducidad o condiciones sanitarias inadecuadas; una forma de riesgo alimentario que siempre existió. Por otro lado, los riesgos modernos están representados por la regulación y aditivos alimentarios como hormonas, conservantes químicos y artificiales; éstos provocan nuevas preocupaciones que no existían en escala tan substancial en los siglos anteriores y son cada vez más difíciles para calcular, prevenir o evitar. La regulación de los riesgos modernos es controversial, ya que ocurren en un escenario de preocupación pública, incerteza científica y desafíos políticos (Buchler, et al., 2010).

Entre algunos de los ejemplos de riesgos tradicionales y modernos que crean inestabilidad en el sistema agroalimentario, se puede mencionar la enfermedad de la Encefalopatía Espongiforme Bovina (conocida como "vaca loca"), la contaminación masiva de Salmonella en el pollo, o más recientemente adulteración con restos de melamina en derivados de leche en polvo, como complemento alimentario para niños, ocurrido en la China. Éste último alcanzando dimensiones internacionales al ser producido por medio de 22 fabricantes y empresas distribuidoras, las cuales vendieron los productos lácteos para otras regiones de Oriente y Occidente.

Los riesgos alimentarios son cada vez más comunes en la contemporaneidad y cualquiera que sea el tipo de riesgo, este puede adquirir implicaciones internacionales impulsadas por la creación de una nueva corriente del sector minoristas que determina y reorganiza el negocio al por menor para aumentar su rentabilidad (Busch, 2007). Estos eventos crean nuevas actitudes de 
inseguridad y desconfianza en el público consumidor; así como preocupaciones sobre el propio desarrollo industrial, en una época que forma parte de la sociedad de riesgo global (Beck, 2002).

\section{Calidad estandarizada: ¿ Una respuesta?}

Las preocupaciones de la sociedad en relación con los riesgos alimentarios, impulsan a nuevos retos y acciones. En un escenario favorable, se esperaría una actitud más proactiva por parte de los consumidores durante sus escogencias de alimentos; a la vez, un sector productor interesado por los riesgos y preocupaciones que afecta a esos consumidores. La calidad en los alimentos es un tema que inquieta a varios sectores, tanto a entidades preocupadas por la salud, como a quienes sufren por las consecuencias económicas que afectan a las corporaciones de las cuales forman parte.

Junto con las preocupaciones alimentarias que acarrea el sistema, ha sido creada una vasta lista de certificaciones de la calidad, las cuales incluyen grados y estándares de medición por medio de los cuales los productos y los entes productores son juzgados. La llamada teoría de las convenciones desarrollada por Boltanski e Thévenot (1991) sugiere una perspectiva de análisis para comprender este proceso de calificación de los productos, e indica que las personas necesitan de un marco analítico (o convención) para juzgar la calidad del producto antes de que estén dispuestas a considerar su compra o venta.

Para la teoría de las convenciones, las reglas no son anteriores a la acción y tampoco son elaboradas fuera de la acción, surgen en el interior del proceso de coordinación de los actores. Son, por lo tanto, representaciones dinámicas de la negociación, y como tales, dependen de la existencia de puntos en común entre los actores involucrados. La calificación de los objetos es por eso, simultáneamente, la calificación de los actores involucrados (Wilkinson, 1999, p. 67).

En la obra de Boltanski y Thévenot los autores indican sobre la existencia de seis mundos diferentes en donde ocurren acciones colectivas entre los actores, y a partir de estas surgen formas de calificar asociadas a diferentes valores: inspiracional (creatividad), doméstico (lealtad/ confianza), opinión (reputación), cívico (representación), mercado (competitividad/ precio), industrial (productividad/ eficiencia) (Boltanski y Thévenot, 1991).

Por otro lado, Pringent y Hérault (2005) establecen que la calidad de los alimentos es un concepto extremadamente enriquecedor, haciendo referencia a dos estudios sobre el tema. El primer estudio es de Cazes (2001, citado en 
Pringent y Hérault, 2005), quien describe diferentes componentes en que la calidad de los alimentos puede ser agrupada, ellos son: calidad nutricional (salud), propiedades funcionales (servicio), calidad organoléptica (placer), calidades sociales (pertenencia), calidad simbólica (cultural), calidad higiénica (seguridad) y calidad humanística (ambiente y valores morales). El segundo estudio que menciona, es de Sylvander, Porin y Mainsant (1998, citados en Pringent y Hérault, 2005), quienes establecen que existen diferencias entre dos tipos de propiedades específicas para considerar la calidad: i) aquellas que se pueden diferenciar por medio de las capacidades sensoriales, que son obtenidas a partir del mismo producto y ii) las que son constatadas por medio de los productores, porque el público consumidor no puede comprobarlas por sí mismo, y que están relacionadas con el tipo y características del proceso del alimento, desde su producción hasta su comercialización.

De acuerdo con lo anterior, es apreciable que existen diferentes perspectivas durante el proceso de calificación de un alimento, no significa que un tipo de valoración se encuentre por encima de otra, sino que existen formas diferentes de medir la calidad. Los estándares de calidad tienen como fin, demostrar e informar al público consumidor acerca de las propiedades del producto. La pluralidad de actores involucrados en el proceso, de cierta forma, justifica la diversidad de certificaciones de la calidad que hoy es posible encontrar en el mercado. Sin embargo, la diversidad de criterios representa también, un desafío en relación con discernir entre lo que significa buena o mala calidad en determinado producto.

A pesar de la importante tarea de las certificaciones y estándares de la calidad para la diferenciación de los alimentos, su proliferación visible a partir de adiciones en los empaques de alimentos, ha generado confusión en los consumidores. Más que un instrumento de mercado, las certificaciones y estándares de la calidad se han convertido en mecanismos para regular los mercados. A la vez, los actores involucrados en el sistema agroalimentario global están desempeñando, cada vez más, un papel en la fijación de las mismas reglas que gobiernan sus actividades, situación que genera nuevas preocupaciones sobre la eficacia y la legitimidad de esas reglas (Goodman y Redclift, 1992).

Según Bush y Bingen (2007) los grados y estándares que se generan para la certificación de la calidad están implicados en decisiones sobre quién podría participar en la toma de decisiones y quién podría producir qué, al igual que en el tema de la justicia distributiva y lo que constituye una buena vida (diferente para unos grupos y otros). Sin embargo, no todo el mundo es admitido 
en las negociaciones que dan lugar a la creación, modificación o mantenimiento de ciertos grados o estándares y estos son producidos por comités técnicos que no siempre prestan atención a las características de los grupos que no se encuentran representados en la mesa de negociación.

Aunque los estándares de la calidad generan las reglas para la entrada en un mercado transparente, también pueden permitir o denegar el acceso a un producto, o excluir a determinado sector. Además, los estándares pueden redistribuir el ingreso, la riqueza, el poder, el estatus y el prestigio (Bush y Bingen, 2007). Por lo tanto, su uso tiene repercusiones importantes en el tema de comercialización, en donde solamente algunos son admitidos, por decisiones que incluso les son desconocidas.

Las certificaciones de calidad de un producto, aunque en la mayoría de los casos se ven reflejadas en distintivos del empaque, pueden resultar en una estrategia limitada para transmitir las características reales de ese producto al público consumidor. Aún así, existen consumidores que responden positivamente a la compra, desconociendo los estándares de calidad que han sido destinados para ese alimento, sin cuestionarse o formar parte de ese proceso de calificación al que son totalmente ajenos.

En el competitivo sistema alimentario existen preocupaciones en el tema del consumo, ya que las compañías de alimentos deben satisfacer a sus accionistas alentando a más personas a comer más de sus productos, por lo que buscan nuevas audiencias entre los miembros de los grupos minoritarios (Nestle, 2013). Además, las corporaciones dominan las cadenas de abastecimiento, exigiendo a los entes proveedores características de variedad y calidad de los productos alimentarios, de tal forma que los modelos de tamaño y forma de empaque, etiquetas de marca y las características organolépticas muchas veces son influenciados y, al mismo tiempo, controlados por los supermercados (Busch, 2007).

Cada día la industria alimentaria propone y ofrece nuevos productos. Lejos de reaccionar al proceso normal de consumo, como una condición permanente de la vida y un aspecto inalienable de ésta, el consumismo se apodera de la población como un atributo de la sociedad. Así, el consumismo ocurre cuando la capacidad individual de querer, desear y alcanzar es separada de los individuos (alienación) para ser una fuerza externa capaz de conducir a la "sociedad de los consumidores". Como indica Bauman (2007), se trata de una "vida del ahorismo", es decir, una sociedad que promete felicidad a cada 
instante y en este caso, por medio de los productos ofertados y promocionados por sus diferentes atributos.

La industria alimentaria implica estandarización, para algunos estudiosos ésta, afecta los métodos de producción y los hábitos de consumo de la población, porque promueve patrones uniformes que muchas veces son protagonizados por las grandes corporaciones multinacionales. Estas corporaciones introducen y difunden pautas estandarizadas de producción en serie, a la vez que promocionan marcas comerciales registradas y reconocidas universalmente (Ruiz, E., Castelló, P., Climent, L., Escalona, A. I., Hernández, M., Loscertales, B., Frutos, L., 2013).

Aunque muchas veces desconocidas en el contexto local, estas certificaciones y estándares de la calidad, representan una importante diferencia para las corporaciones de alimentos, que les permite competir en el mercado global, en donde existe una producción estandarizada, homogénea y en masa. Por lo tanto, un distintivo que certifique la calidad puede hacer que ese alimento represente una diferencia para el público consumidor, frente a una exorbitante cantidad de productos, y por consecuencia, una ventaja importante para un determinado sector de la industria alimentaria.

Ante este panorama de la modernización, surgen cuestionamientos acerca de la participación en los procesos de estandarización homogéneos que caracterizan al mercado dominante. Ese proceso implica una progresiva exclusión de la dimensión humana relacionada al conocimiento práctico local, para privilegiar una perspectiva centrada exclusivamente en la eficiencia técnica, y se dejan a un lado los valores de la creatividad, la lealtad, la confianza, reputación y representación; por otros más orientados a la competitividad, el precio, la productividad y eficacia de los alimentos, de acuerdo con las valoraciones de la calidad descritas por Boltanski y Thévenot (1991) y mencionadas anteriormente.

\section{Las perspectivas de la calidad y las ferias del agricultor}

Si bien los distintivos para identificar la calidad de los alimentos están en desarrollo constante, las nuevas formas de comercio entre el sector productor y consumidor también están siendo impulsadas simultáneamente. Las ventas directas, los mercados de proximidad o locales y los circuitos cortos, demuestran que los sujetos participantes están siguiendo una tendencia convergente que no puede simplemente ser considerada como una transacción económica (Prigent y Hérault, 2005). En este sentido Darolt (2013) menciona que la 
crisis del modelo agroalimentario dominante abre espacio para la discusión de nuevas preposiciones de desarrollo local, que incorporan variables técnico-productivas, económicas y ambientales, pero también los valores sociales, éticos y culturales.

En la modernidad, el sistema alimentario hegemónico y los mercados alternativos no constituyen circuitos económicos completamente separados, cada uno de ellos modela al otro y a menudo se superponen de forma significativa (Pratt, 2009). Este proceso atañe también al tema de certificaciones de la calidad, en donde cada vez es más común encontrar estrategias que buscan diferenciar los productos en los mercados alternativos. Según Pratt (2009), las etiquetas de "orgánico", "comercio justo" o "local" revelan mucho sobre el grado en que sus grupos productores han resistido o han sido absorbidos por los intereses de las empresas de la industria alimentaria.

Cuando se considera la calidad, cada producto y cada situación, exige un análisis particular. El tipo de mercado, el tipo de instituciones, los saberes y las técnicas utilizadas, las normas jurídicas que conciernen a los productos y los recursos naturales condicionarán el proceso de calificación (Muchnik, 2006). Por lo tanto, es diferente la valoración de la calidad al tratarse de un proceso de compra a través de una cadena larga de comercialización, como el caso de los supermercados, o si se trata de un mercado local, como las ferias del agricultor.

La sociología del consumo analiza ese proceso centrando sus intenciones en el sentido de cómo los valores (calidades) son formados en universos relacionales. Muchos estudios abarcan la capacidad de los movimientos de los grupos consumidores en recontextualizar los alimentos, imprimiéndoles significados distintos de aquellos que "originalmente" portaban (Friedman, 1999; Stolle, Hooghe e Micheletti, 2005). De esta situación, emerge una discusión particularmente importante sobre el público consumidor-ciudadano y las formas de "consumo politizado" con sus prácticas de boycott y buycott. Miller (2007) analiza la dimensión política de ese proceso, sugiriendo que, al seguir las trayectorias de los bienes, es posible "desfetichizarlos" en la medida en que se revelan las relaciones humanas que sustentan sus imágenes.

Por su parte Campbell (2005) construye una noción de craft consumer para analizar cómo, en el acto de consumir, los sujetos consumidores transforman mercadurías en objetos personalizados. En esta misma perspectiva, Allaire (2004) sustenta que un producto altera sus calidades como resultado de las redes en donde circula. Nuestra premisa sugiere que las ferias de agricultores 
alteran la trayectoria social de los alimentos, asociándolos a otros bienes y valores, y produciendo experiencias singulares de producción y consumo.

Para analizar el tema de perspectivas de la calidad en el nivel local, se presenta el caso de las ferias del agricultor como un mercado local que constituye un circuito corto de comercialización en Costa Rica. En este caso, se incluirá, en el término de circuito corto, aquellos que movilizan hasta un máximo de un intermediario entre productor y consumidor (Chaffotte y Chiffoleu; 2007, mencionado por Darolt, 2013). En el caso particular de las ferias del agricultor, existe principalmente una venta directa entre sujeto consumidor y sujeto productor, aunque se ha evidenciado la presencia de entes intermediarios (prohibido en la Ley 8533 que las regula), en este caso, un actor más dentro de ese circuito, aún se considera bastante diferenciado de las grandes cadenas de supermercados que representan otro segmento.

El Programa Nacional de Ferias del Agricultor fue creado en el año 1978 como:

Programa de mercadeo de carácter social, de uso exclusivo para los pequeños y medianos productores nacionales de los sectores de la producción agrícola, pecuaria y forestal, pesca y acuicultura, avicultura, agroindustria y artesanía, en forma individual u organizada, con el objeto de poner en relación directa a consumidores y productores, de manera tal que los primeros obtengan mejor precio y calidad, y los segundos incrementen su rentabilidad, al vender de modo directo al consumidor (Ley N. $\left.{ }^{\circ} 8533,2006\right)$.

Según la Organización de las Naciones Unidas para la Alimentación y la Agricultora (FAO por sus siglas en inglés, 2011) en Costa Rica, las ferias del agricultor cuentan con una gran diversidad cultural y representativa de las diferentes regiones del país, conformando espacios interculturales. Este concepto se refiere a la comunicación, el respeto entre los colectivos culturales y el reconocimiento de sus hábitos y costumbres. Además, en las ferias existe la participación y el empoderamiento de los hombres y mujeres representantes de esos colectivos, visibilizando a grupos productores agropecuarios o artesanos y que comercializan en dicho mercado.

Actualmente las ferias del agricultor se expanden por todo el territorio nacional cada fin de semana, concentrando a grupos productores y consumidores de diversas regiones del país en diferentes barrios urbanos, tanto dentro de la Gran Área Metropolitana como fuera de ella. Aunque existen aspectos por mejorar, las ferias del agricultor representan una experiencia exitosa desde 
la propuesta de mercados alternativos, pues permiten un espacio viable de comercialización y generación de ingresos (no solo en términos monetarios sino también de alimentos en espacie) para las familias agricultoras, en un ambiente que promueve la compra directa, contribuyendo con la SAN de esas familias y las consumidoras (Zúñiga y Jensen, 2013).

De acuerdo con las encuestas realizadas durante el 2000 al 2015 por el Programa Integral de Mercadeo Agropecuario (PIMA, 2016), institución que administra el Centro Nacional de Abastecimiento y Distribución de Alimentos (CENADA) en Costa Rica, las ferias del agricultor siguen siendo el mercado favorito de compra de frutas y hortalizas $(21,2 \%)$, principalmente por la calidad y los precios que ofrecen; seguido de las verdulerías independientes $(19,6 \%)$ y de las cadenas de supermercados (18,8\%). Así, la calidad y el precio figuran dentro de las razones principales del por qué los hogares costarricenses se abastecen en esos establecimientos, seguidos de otras razones de importancia como la cercanía de sus hogares, la variedad de productos (PIMA, 2016). A pesar de esta tendencia, los alimentos comercializados en las ferias del agricultor, en su mayoría, no cuentan con distintivos que informen acerca de alguna certificación de la calidad. Sin embargo, es posible reconocerla a partir de otras formas, pues se identifica a la alimentación como un hecho social, que adquiere un interés creciente en el periodo actual, cuando se cuestionan los modelos de desarrollo agrícola basados en la producción en masa y la estandarización. En este sentido, Muchnik (2006) indica que "las personas en sociedad integran la alimentación como componente en la construcción de sus identidades".

En el tema alimentario no es posible aislar los factores biológicos de los factores sociales, y en el acto de comer se pone en juego variables como la composición y las características de los alimentos, la salud del sujeto consumidor, su identidad cultural, la dinámica de los territorios donde habita, la evolución de la producción y de los grupos productores (Muchnik, 2006). Estos elementos son considerados de diferente forma durante su comercialización y podrían ser valorados o no por los consumidores. Así, y de acuerdo con las valoraciones desarrolladas por Boltanski y Thévenot (1991), del mundo industrial y de mercado -de las cuales no escapan los mercados locales-, en donde existen valores de competitividad, precio, productividad y eficiencia; en las ferias del agricultor toman fuerza los valores presentes del mundo inspiracional, doméstico, de opinión y cívico que se traducen en creatividad, lealtad, confianza, reputación y representación. 
En la sociología económica, existen varios estudios que han desarrollado el término "embeddedness". Desde su introducción por Polanyi (1957) hasta el artículo fundacional de Granovetter (1985), este término se refiere a un enfoque de arraigo en que las redes sociales- basado en gran medida por la confianza- sostienen las relaciones económicas y las instituciones. Se agrega además, que la mayoría del comportamiento está estrechamente integrado en redes de relaciones interpersonales y que las relaciones sociales son fundamentales para los procesos de mercado. Esas relaciones sociales son características en las ferias del agricultor, que desde la perspectiva y los tipos de calidad mencionados por Sylvander, Porin y Mainsant (1998), pueden visibilizarse como atributos que constituyen la calidad en este mercado local. Así, al considerar las calidades sociales, los autores hacen referencia a la pertenencia, la calidad simbólica de contenido cultural y la calidad humana que contempla valores de ambiente y morales.

La frescura del alimento juega un papel importante en el caso de la calidad nutricional, que responde a la manera en que son satisfechas las necesidades nutricionales del organismo y la existencia de elementos que puedan afectar su digestión; así como la calidad organoléptica, que contempla las propiedades verificables sensorialmente (visuales, olfativas, gustativas, de placer y plenitud, entre otras). Si bien es cierto, los alimentos vendidos en las ferias del agricultor generalmente han sido cosechados durante la semana y la mayoría de ellos el día anterior a su venta; también es importante ser conscientes sobre la intermediación y las técnicas de producción que realizan los agricultores y agricultoras. Así, en la feria es posible encontrar productos agroecológicos u orgánicos a precios accesibles para el consumidor; pero, también productos sometidos a técnicas convencionales y uso excesivo de agroquímicos que atentan contra la salud. Algunas de estas prácticas podrían estar siendo utilizadas de forma consciente y otras, por el contrario, fuera de los límites sanitarios requeridos.

En este sentido, el consumidor cumple un papel fundamental durante el proceso de compra, el hacer efectivo su derecho a una alimentación adecuada y saludable. En la medida en la que los consumidores puedan conocer quién produce, de dónde viene ese alimento y cómo ha sido su tratamiento desde que fue cultivado hasta su venta, reafirman su papel como consumidores responsables y participan de los procesos de certificación de la calidad. Kneafsey et al. (2008) argumentan sobre la desconexión de los grupos consumidores, en cuanto a que la mayoría sabe muy poco acerca de dónde provienen sus alimentos, de qué están hechos, cómo son producidos y por quién. Para el 
consumidor, el reconocimiento sobre estas características del alimento, puede ser identificado con mayor facilidad durante la compra directa, en relación con la compra a un detallista, sobre todo porque se favorece la reconexión entre productor y consumidor.

Sobre la calidad higiénica, cuyo objetivo es garantizar la salubridad de los alimentos y encontrarse libre de contaminantes, la Ley 8533 que regula las ferias del agricultor, establece ajustarse a las normas vigentes que contempla la Ley General de Salud en Costa Rica, y los grupos agricultores deberán portar el carné de manipulación de alimentos al día. Aun así, existe la necesidad de desarrollar acciones de mejoramiento en su infraestructura (FAO, 2011) para lograr condiciones óptimas durante la venta, así como el monitoreo constante de las prácticas de higiene e inocuidad que desarrollan los grupos productores y consumidores, pues todos forman parte del proceso que conlleva alimentarse.

Los tipos de calidades mencionados amplían el foco de atención del consumidor cuando realiza su compra y a la vez, exige de éste un compromiso y participación de la valoración de la calidad del alimento que va a consumir. Sin embargo, los criterios de preferencia de compra del público consumidor estarán también determinados, tanto por el precio y la disponibilidad del alimento, como también por la información que tenga sobre éste (Muchnik, 2006). En ese sentido, la obtención de información sobre el alimento comprado en una feria del agricultor va a depender, principalmente, de la relación que exista entre consumidor y productor durante la compra. A pesar de que se trata de un acto cotidiano en este espacio de mercado, es necesario valorar la importancia de estas relaciones sociales durante la compra de un alimento, de forma que, el público consumidor logre tener mayor criterio para confiar, exigir o elegir calidad del alimento durante su compra.

Los procesos de enraizamiento, confianza y de lo local son elementos clave para valorar la calidad de un alimento (Goodman, 2003). Cuestionar las formas de certificación de la calidad y el tipo de estándares que están siendo valorizados, según el mercado en donde se realiza la compra del alimento, sugiere atender criterios más amplios. Este proceso, implica que como consumidores seamos reflexivos en cuanto a la representatividad acerca de lo que es de buena o mala calidad, así como en relación con la pérdida de identidad en la estandarización. Además, es importante ser consientes sobre las diferentes formas de comercializar, permitiendo dar valor a las ferias del agricultor que se encuentran inmersas en un reordenamiento del sistema agroalimentario. Tal sistema, es orientado a creer que existe una única lógica de calificar los alimentos, en donde la 
dimensión humana por medio del establecimiento de relaciones sociales, parece no tener espacio aun cuando juega un papel primordial.

\section{Consideraciones finales}

Las diferentes perspectivas de la calidad y las características de los mercados, posibilitan o no la participación del consumidor en el proceso de valoración que define lo que es un alimento de buena o mala calidad. En el sistema dominante existen estándares de calidad que muchas veces son desarrollados por un reducido grupo de actores, que no necesariamente representa a los diversos sectores. Además, su establecimiento puede excluir a aquellos alimentos y mercados que no cumplen con sus requisitos, pero que representan valores diferenciados de la calidad que tienden a ser invisibilizados por el mundo comercial y la perspectiva construida por el sistema hegemónico.

Paralelo al sistema dominante caracterizado por el sector detallista, los mercados locales y en particular las ferias del agricultor, son una alternativa importante para la compra de alimentos que favorece la seguridad alimentaria y nutricional de muchas familias. En ellas, más allá de la comercialización, se genera un espacio favorecido por las relaciones sociales, que generan proximidad entre productores y consumidores, posibilitando así el hecho de que las personas elijan, compren, reclamen, valoren, exijan, apoyen, promuevan $y$, en fin, interactúen como parte del proceso de valoración de la calidad de alimentos, del cual nunca serán participantes en el imperio alimentario. Para finalizar, retomamos las palabras de Beck (1997, p. 209): "Entre más se modernizan las sociedades, más agentes adquieren la capacidad de reflexionar sobre sus condiciones sociales de existencia y de cambiar".

\section{Referencias}

Allaire, G. (2004). Quality in economics: a cognitive perspective. In M. Harvey, A. McMEEKIN, A. WARD (Eds.), Qualities of food (pp. 66-92). Manchester: Manchester University Press

Bauman, Z. (2007). Vida de consumo: a transformação das pessoas em mercadoria (Cap. 1). Rio de Janeiro: Zahar.

Beck, U. (1997). "La reinvención de la política: hacia una teoría de la modernidad reflexiva“, en BECK, U.; GIDDENS, A.; LASH, S. Modernización reflexiva. Política, tradición y estética en el orden social moderno. Madrid: Alianza Universidad. p. 13-74. (Original en Inglés: BECK, U. “The reinvention of politics: Towards a Theory of reflective modernization”, 
in BECK, U.; GIDDENS, A.; LASH, S. Reflective modernization. Politics, Tradition and Aesthetics of the Modern Social Order. Cambridge: Polity Press, 1994.)

Beck, U. (2002). La sociedad del risco global. Madrid: Sieglo XXI Espana Editores.

Beck, U.; Giddens, A.; Lash, S. (1994). Reflective modernization. Politics, Tradition and Aesthetics Boström, M.; Klintman, M. (2008). Ecostandards, product labelling and green consumerism. Hampshire: PalgraveMacmillan.

Boltanski, L., Thevenot, L. (1991). De la justification. Paris: Gallimard.

Buchler, S., Smith, K., Lawrence, G. (2010) Food risks, old and new.Demographic characteristics and perceptions of food additives, regulation and contamination. In Australia. Journal of Sociology, 46(4), 353-374.

Bush, L; Bingen, J. (2007). Introduction: a new world of standards. In: BINGEN, Jim; BUSH, Lawrence (Eds.). Agricultural standards: the shape of the global food and fiber system. Dordrecht, Holland: Springer, 2007. p. 3-28.

Busch, L. (2007). Performing the economy, performing science: From neoclassical to supply chain models in the agrifood sector. Economy and Society, 36(3), 439-468.

Campbell, C. (2005). The craft consumer. Journal of Consumer Culture, 5(1), 23-42.

Darolt, M.R. (2013). Circuitos curtos de comercialização de alimentos ecológicos: reconectando produtores e consumidores. In: P. Niederle, L. Almeida and F. Vezzani (orgs.) Agroecologia: práticas, mercados e políticas para uma nova agricultura. 1ed. Curitiba. ISBN No 978-85-63806-16-1

Friedmann, H. (2016). Food Regime Analysis and Agrarian Questions: Widening the Conversation. Coloquium Global governance/politics, climate justice \& agrarian/social justice:linkages and challenges. ISS. Recuperado de: https://www.iss.nl/fileadmin/ASSETS/iss/Research_ and_projects/Research_networks/ICAS/57-ICAS_CP_Friedman.pdf

Friedman, M. (1999). Consumer boycotts. New York: Routledge.

Goodman, D. (2003). The quality 'turn' and alternative food practices: reflections and agenda. Journal of Rural Studies, 19, p.1-7. 
Goodman, D.; Dupuis, E. M.; Goodman, M. (2012). Alternative Food Networks: knowledge, practice and politics. London: Routledge. (Parte I Alternative food networks: reflexivity and shared knowledge practice).

Goodman, D., Redclift, M. (1991) Refashioning nature: food, ecology and culture (Cap. 3 e 6). London: Routledge.

Granovetter, M. (1985). Economic Action and Social Structure: The Problem of Embeddedness," American Journal of Sociology. 91(3). pp 481-510.

Instituto de Nutrición de Centroamérica y Panamá (INCAP). (2018) Acerca de SAN. Página oficial. Recuperado en: http://www.incap.int/index. php/es/acerca-de-san

Kneafsey, Cox, Holloway, Dowler, Venn y Tuomainen. (2008). Reconnecting Consumers, Producers and Food (Chapter 1, pp. 1-25). Oxford/UK. Berg Editorial Officer.

LEY N. ${ }^{\circ} 8533$. (18 de agosto de 2006). La Gaceta N. ${ }^{\circ} 159$ de la República de Costa Rica, San José, Costa Rica.

Miller, D. (2007). Consumo como cultura material. Horizontes Antropológicos, 13(28), 33-64.

Nestle, M. (2013). Food Politics: How the Food Industry Influences Nutrition and Health. Berkeley: University of California, 2013, 3(Introduction), pp. 21-48.

McMichael, P., 2016. Food Regime for Thought. Coloquium Global governance/ politics, climate justice \& agrarian/social justice:linkages and challenges. Recuperado en: https://www.iss.nl/fileadmin/ASSETS/iss/Research_and_ projects/Research_networks/ICAS/56-ICAS_CP_McMichael.pdf

Muchnik, J. (2006). Identidad territorial y calidad de los alimentos: Procesos de calificación y competencias de los consumidores. Agroalim, Mérida.11 (22). Pp 89-98, jun. Recuperado de <http://www.scielo.org.ve/ scielo.php?script=sci_arttext\&pid=S1316-

Organización de las Naciones Unidas para la Alimentación y la Agricultura (FAO). (2011). Ferias del agricultor, un espacio intercultural por excelencia. Programa conjunto: Políticas interculturales para la inclusión y generación de oportunidades. Recuperado de http://nacionesunidas. or.cr/dmdocuments/Sistematizacion.pdf 
Ploeg, J. (2008). Camponeses e Impérios Alimentares. Lutas por autonomia e sustentabilidade na era da globalização. Porto Alegre: Editora UFRGS.

Polanyi, M, (1958) Personal Knowledge: Towards a Post-Critical Philosophy. University of Chicago Press. ISBN 0-226-67288-3

Polanyi, K. A. (1980). A grande Transformação. As origens da nossa época. RJ: Campus.

Prigent-Simonin, A. H; Hérault-Fournier, C. (Mai, 2005). The role of trust in the perception of the quality of local food products: with particular reference to direct relationships between producer and consumer. Anthropology of food, 4. Recuperado de http://aof.revues.org/document204.html

Pratt, J. (2009). Incorporation and Resistance: Analytical Issues in the Conventionalization Debate and Alternative Food Chains. Journal of Agrarian Change, 9(2), 155-174.

Programa Integral de Mercadeo Agropecuario (PIMA). (2013). Tendencias de consumo de frutas, hortalizas, pescado y mariscos en las familias de Costa Rica. Recuperado de file://C:/Users/Seguran\%C3\%A7a/Downloads/ Documento\%20Estudio\%20Tendencias\%20Consumo\%202013-web.pdf

Ruiz, E., Castelló, P., Climent, L., Escalona, A. I., Hernández, M. ; Loscertales, B., Frutos, L. M. (2013). La calidad del vino a la luz de la teoría de las convenciones. Aplicación a las denominaciones de origen aragonesas. Estudios Geográficos, LXXDIV(274), 231.254. Recuperado de http://estudiosgeograficos.revistas.csic.es/index.php/estudiosgeograficos/article/viewFile/397/397

Stolle, D., Hooghe, M. and Micheletti, M. (2005). Politics in the supermarket. International Political Science Review, 26, 245-269.

Sylvander, B., Porin. F., Mainsant. P. (1998). “Les facteurs de succès dans l’agro alimentaire", VII journées des Sciences du muscle et technologies de la viande, Rodez, 14

Wilkinson, J. (1999). A contribuição da teoria francesa das convenções para os estudos agroalimentares: algumas considerações iniciais. Ensaios FEE, Porto Alegre, 20(2), 64-80.

Zúñiga M y Jensen, M. (2013). Conocimiento en SAN de los agricultores participantes de las ferias del agricultor, una base para fortalecer sus capacidades. Memoria del III Congreso de Desarrollo Local en La Habana, Cuba. 